\title{
C1QTNF6 is overexpressed in gastric carcinoma and contributes to the proliferation and migration of gastric carcinoma cells
}

\author{
HAI-XIA QU ${ }^{1}$, LIN CUI ${ }^{2}$, XIN-YING MENG ${ }^{2}$, ZHI-JIE WANG $^{3}$, YAN-XIN CUI $^{2}$, \\ YUN-PENG YU ${ }^{2}$, DONG WANG ${ }^{3}$ and XIANG-JUN JIANG ${ }^{1}$ \\ ${ }^{1}$ Department of Gastroenterology, Qingdao University Affiliated Qingdao Municipal Hospital; \\ ${ }^{2}$ Department of Gastroenterology, Qingdao Municipal Hospital, Qingdao, Shandong 266071; \\ ${ }^{3}$ Digestive Endoscopy Center, Department of Gastroenterology, Changhai Hospital, Shanghai 200433, P.R. China
}

Received June 15, 2018; Accepted October 30, 2018

DOI: $10.3892 /$ ijmm.2018.3978

\begin{abstract}
In the present study, proteins differentially expressed between gastric cancer tissue and para-tumoral normal gastric tissues were screened, and the function of the highly expressed protein C1QTNF6 in gastric carcinoma was investigated. The differential expression of mRNAs extracted from the tumor and adjacent tissues was analyzed using GeneChip assay. An AGS si-C1QTNF6 cell line was constructed using shRNA-C1QTNF6 lentivirus. The cell invasion and migration ability of C1QTNF6-knockdown cells were determined by Transwell chamber migration and wound healing assays, respectively. The effects of C1QTNF6 on AGS cell cycle distribution and apoptosis were detected using a FACScan flow cytometer. The results demonstrated that the expression of 109 genes was increased and the expression of 129 was decreased in tumor tissues. Among these genes, the C1QTNF6 gene was highly expressed in tumor tissues and the AGS7901 cell line. C1QTNF6-knockdown decreased the cell growth, and the proliferative and migration ability, as well as increasing the apoptosis of gastric carcinoma cells. In addition, the number of AGS cells in the G2/M phase was significantly increased after 5 days of C1QTNF6-shRNA lentivirus infection. The results of the present study indicated that C1QTNF6 serves an important role in the development of gastric carcinoma. C1QTNF6 is involved in promoting the proliferation and migration, and in reducing the apoptosis of
\end{abstract}

Correspondence to: Professor Xiang-Jun Jiang, Department of Gastroenterology, Qingdao University Affiliated Qingdao Municipal Hospital, 5 DongHai Zhong Road, Qingdao, Shandong 266071, P.R. China

E-mail: drjxj@163.com

Professor Dong Wang, Digestive Endoscopy Center, Department of Gastroenterology, Changhai Hospital, 168, Changhai Road, Shanghai 200433, P.R. China

E-mail: dongwang0901@sina.com

Key words: C1QTNF6, AGS7901 cell line, gastric carcinoma gastric carcinoma cells. These results provided a potential therapeutic target for the treatment of gastric carcinoma.

\section{Introduction}

Gastric carcinoma (GC) is the fourth most prevalent type of cancer and is the second highest contributor to global cancer-related mortality (1). GC remains the second most common cause of cancer-associated mortality and health problems in China, despite the lower incidence of GC observed over the past several years (2). Although gastric cancer can be surgically excised and inhibited by chemotherapy, gastric tumor cells continue to grow and metastasize elsewhere in the body, which leads to a poor prognosis. Uncontrolled tumor cell proliferation and migration are two important features of GC. Therefore, inhibition of cancer cell proliferation and migration, and promotion of cancer cell apoptosis are promising therapeutic targets for GC. Investigation of the pathological characteristics of GC and associated mechanisms will provide novel insights for the discovery of potential therapeutic targets.

C1q and tumor necrosis factor superfamily are involved in several biological processes, including inflammation, apoptosis and cell differentiation. C1q/tumor necrosis factor-related proteins (CTRP proteins) have been revealed to serve a role in carcinogenesis and cancer progression (3). The C1q/TNF-related protein family is comprised of 16 CTRP members, CTRP1-9, 9B, 10-15. Among these, CTRP3, CTRP4 and C1QTNF6 have been revealed to be associated with tumor promotion. All CTRP members are secreted proteins, and are widely expressed in various tissues and cell types (4-8). CTRP4 was demonstrated to function as a tumor-promoting inflammatory regulator, and to promote tumor cell survival and reduce drug-induced apoptosis (3). These findings strongly suggested that CTRP4 is a potential therapeutic target. CTRP8 was reported to be involved in brain cancer (9). It was also demonstrated to enhance motility and matrix invasion by human glioblastoma cells (10). CTRP8-induced migration of human glioma cells was revealed to be inhibited by a small competitor peptide derived from C1QTNF6 (11). Western blotting experiments have demonstrated that C1QTNF6 is highly expressed in human 
hepatocellular carcinoma tissues. An immunohistochemistry assay indicated that C1QTNF6 is mainly localized in hepatocellular carcinoma cells and endothelial cells in tumor tissues. High expression of C1QTNF6 was revealed to activate the Akt signaling pathway, increase tumor angiogenesis and reduce the necrosis of HepG2 cells (12). C1QTNF6-interference was revealed to inhibit the Erk1/2 signaling pathway in 3T3-L1 adipocytes (13). C1QTNF6 was also revealed to serve as an endogenous complement regulator that exhibited a prominent therapeutic effect in arthritis (14). It was also demonstrated that C1QTNF6 inhibited fibrogenesis by TGF- $\beta 1$-stimulated human dermal fibroblasts (15).

The present study investigated the expression and localization of C1QTNF6 in GC specimens. Furthermore, the pathological functions of C1QTNF6 in GC, including cell growth, proliferation, cycle, migration and apoptosis, were studied by transfection of AGS cells with lentivirus expressing siRNA-C1QTNF6.

\section{Materials and methods}

Cell culture and regents. All cell lines were purchased from American Type Culture Collection (American Type Culture Collection, Manassas, VA, USA). SGC-7901, AGS, BGC-823 and MGC-803 cells were cultured in Dulbecco's modified Eagle's medium (DMEM; Invitrogen; Thermo Fisher Scientific, Inc., Waltham, MA, USA), supplemented with $10 \%$ fetal bovine serum (FBS) and 1\% penicillin/streptomycin in $5 \% \mathrm{CO}_{2}$ at $37^{\circ} \mathrm{C}$. Cell growth was monitored using Celigo instrument (Nexcelom Corporation, Lawrence, MA, USA). The siRNA targeting C1QTNF6 (target sequence site: TGTGTGAGATCCCTATGGT) was purchased from Shanghai GeneChem (Shanghai, China). SYBR-Green quantitative polymerase chain reaction (qPCR) master mix was obtained from Takara Biotechnology Co., Ltd., Dalian, China (cat. no. DRR041B). BrDU kit was obtained from Roche Diagnostics, Basel, Switzerland. FITC-Annexin V apoptosis detection kit was purchased from eBioscience; Thermo Fisher Scientific, Inc. (cat. no. 88-8007).

Patient selection. A total of 64 patients (34 males and 18 females) aged between 27 and 85 years (mean, 62.8 years) undergoing surgical resection of primary GC between March 2012 and June 2015 at the Qingdao Municipal Hospital (Qingdao, China) were selected for the present study. Gene expression profiles were generated from gastric biopsy specimens of 12 patients using GeneChip. Immunohistochemical analysis for C1QTNF6 was performed on the tumors and non-neoplastic gastric mucosal specimens (located $>2 \mathrm{~cm}$ away from the margins of the tumor) obtained from the other 52 patients. None of the patients received chemotherapy or radiation therapy prior to surgery. The pathological diagnosis was made by 2 pathologists based on the depth of infiltration, differentiation, lymph node metastasis, microvascular invasion and nerve invasion (Table I). The Institutional Review Board at the Qingdao Municipal hospital approved the study protocol, and all patients provided written informed consent.

Gene microarray analysis. Total RNA samples were extracted from tumor and peri-tumoral normal gastric tissues
Table I. Pathological characteristics of gastric carcinoma $(\mathrm{n}=52)$.

\begin{tabular}{lcc}
\hline Pathological characteristic & Number & $\%$ \\
\hline Differentiation & 2 & 3.8 \\
Well & 11 & 21.2 \\
Moderate & 39 & 75 \\
Poor & & \\
Depth & 11 & 21.1 \\
$\quad$ Mucosa and submucosa & 7 & 13.5 \\
Muscularis & 34 & 65.4 \\
Serosa & & \\
Lymph node metastasis & 38 & 73.1 \\
$(+)$ & 14 & 26.9 \\
$(-)$ & & \\
Micro vessel invasion & 30 & 57.7 \\
$\quad(+)$ & 22 & 42.3 \\
$(-)$ & & \\
Nerve invasion & 30 & 57.7 \\
$\quad(+)$ & 22 & 42.3 \\
$(-)$ & & \\
\hline
\end{tabular}

using TRIzol reagent (Thermo Fisher Scientific, Inc.). RNA microarray was conducted by Shanghai GeneChem. In brief, the RNA was reverse transcribed at $37^{\circ} \mathrm{C}$ for $60 \mathrm{~min}$ and then at $95^{\circ} \mathrm{C}$ for 5 min using the GeneChip IVT Express kit (Agilent Technologies, Inc., Santa Clara, CA, USA). The product from each reverse transcription reaction was pre-amplified and then the RNA expression was profiled using human GeneChip primeview array (Affymetrix; Thermo Fisher Scientific, Inc.), according to the manufacturer's protocol.

Reverse transcription ( $R T)-q P C R$ analysis. Total RNA was extracted from cells and tissues using TRIzol reagent (Invitrogen; Thermo Fisher Scientific, Inc.), according to the manufacturer's protocol. The RNA was reverse transcribed at $42^{\circ} \mathrm{C}$ for 60 min using the SYBR-Green master mix in a $20-\mu 1$ reaction. The primer sequences were as follows (designed and synthesized by Shanghai GeneChem): GAPDH forward, 5'-TGACTTCAACAGCGACACCCA-3' and reverse, 5'-CACCCTGTTGCTGTAGCCAAA-3'; and C1QTNF6 forward, 5'-GAAAGGGTCTTTGTGAACCTT GA-3' and reverse, 5'-CTGCGCGTACAGGATGACAG-3'. The qPCR reaction cycle was performed using SYBR green master mix as follows: $10 \mathrm{~min}$ at $95^{\circ} \mathrm{C}$ and 40 cycles of $15 \mathrm{sec}$ at $95^{\circ} \mathrm{C}$ and $60 \mathrm{sec}$ at $60^{\circ} \mathrm{C}$. Quantification for each sample was performed using the $2^{-\Delta \Delta C q}$ method (16).

Immunohistochemistry (IHC). Following the tissues being fixed in $10 \%$ formalin for $24 \mathrm{~h}$ at room temperature and embedded in paraffin, sections from tumor and peri-tumoral normal gastric tissues were deparaffinized in xylene (15 min at room temperature, twice), rehydrated in a descending alcohol series (100\%, 5 min twice; 95, 85 and 75\%, 2 min each), and washed in distilled water. Prior to antigen retrieval, 
the tissues were treated with sodium citrate buffer ( $\mathrm{pH}$ 6.0) at $100^{\circ} \mathrm{C}$ for $20 \mathrm{~min}$, and endogenous hydrogen peroxidase activity was inhibited by $0.3 \%$ hydrogen peroxide. Following microwave antigen retrieval at $95-100^{\circ} \mathrm{C}$, the sections were incubated with $5 \% \mathrm{FBS}$ for $10 \mathrm{~min}$ at room temperature to inhibit non-specific staining. Next, the slides were incubated overnight with anti-C1QTNF6 antibody (1:1,000; ab36900; Abcam, Cambridge, UK) in a moist chamber at $4{ }^{\circ} \mathrm{C}$, followed by incubating with anti-rabbit immunoglobulin $\mathrm{G}$ as secondary antibody $(1: 2,000$; ab205718; Abcam) at room temperature for $1 \mathrm{~h}$. The antigen was visualized using a light microscope (magnification, $\mathrm{x} 200$ or $\mathrm{x} 400$ ).

Construction of AGS si-CIQTNF6 cell line. AGS cell lines stably expressing siRNAs were generated by transduction of $5 \mu \mathrm{g}$ of GV115-si-C1QTNF6 or empty vector (Shanghai GeneChem) in a $100 \mathrm{ul}$ transfection mix and $10 \mu \mathrm{l}$ lipofectamine 2000 (Invitrogen; Thermo Fisher Scientific, Inc.) to $1 \mathrm{ml}$ of medium without serum. At $6 \mathrm{~h}$ following transfection, full medium was used for cell culture. Stable knockdown of the gene was determined by RT-qPCR.

Cell cycle assay. Cells were plated in 6-cm culture plates and grown to $80 \%$ confluence. Subsequently, the cells were harvested by centrifugation at $179 \mathrm{x} \mathrm{g}$ for $5 \mathrm{~min}$, washed twice with PBS, fixed overnight with $75 \%$ cold ethanol at $4^{\circ} \mathrm{C}$ and stained with $0.05 \mathrm{mg} / \mathrm{ml}$ propidium iodide (PI) solution (Sigma-Aldrich; Merck KGaA, Darmstadt, Germany) containing $2.5 \mu \mathrm{g} / \mathrm{ml}$ RNase A for $40 \mathrm{~min}$ at $37^{\circ} \mathrm{C}$. The cell cycle distribution was detected using a FACScan flow cytometer (EMD Millipore, Billerica, MA, USA) and the data was analyzed with FlowJo software 10.5.2 (Tree Star, Inc., Ashland, OR, USA).

Wound healing assay. Cells $\left(3 \times 10^{4}\right)$ were seeded onto 96-well plates and grown to $90 \%$ confluence. Next, the cell culture DMEM medium was changed to DMEM containing 0.5\% FBS, and cells were scratched with a 96 Wounding Replicator (V\&P Scientific, Inc., San Diego, CA, USA; cat. no. VP408FH) to create a mechanical wound. Images were obtained at 0,8 and $24 \mathrm{~h}$ using a phase-contrast microscope (magnification, x100). The distance of wound closure was calculated and the associated P-values calculated.

Transwell chamber invasion assay. Transwell chamber invasion assay was conducted using a Transwell kit (Corning Incorporated, Corning, NY, USA), as previously described (17). In brief, following infection with shRNA lentivirus for 3 days, the cells were harvested and suspended at a density of $1 \times 10^{5}$ cells $/ \mathrm{ml}$ in serum-free DMEM medium. A total of $100 \mu \mathrm{l}$ cells were placed in the upper Matrigel-coated chambers, and $600 \mu \mathrm{l}$ DMEM medium containing $30 \%$ FBS was placed in the lower chambers. Additionally, cell suspension containing 5,000 cells was seeded into 96 -well plates, and was subsequently used for measurement of OD570. After $24-\mathrm{h}$ incubation at $37^{\circ} \mathrm{C}$, the non-invaded cells were removed using a cotton swab, while the invaded cells were stained with 2-3 drops of Giemsa solution for 3-5 min at room temperature. The invaded cells on the lower surface of the membrane filter were counted under an inverted microscope (magnification, x100). The data are presented as the mean number of migratory cells per field.

Colony assay. Cells (400-1,000 cells/well) were seeded into 6 -well plates with three replicates. A total of 14 days after seeding, the cells were washed with PBS and fixed with $4 \%$ paraformaldehyde for 30-60 min at room temperature. The fixed cells were stained with Giemsa dyes for 10-20 min at room temperature. The colonies were visualized and counted using an inverted microscope (magnification, x100; Shanghai Caikon Optical Instrument Co., Ltd., Shanghai, China; cat. no. XDS-100).

Proliferation assay. Cell proliferation was assessed by MTT assay or with the use of Roche BrDU kit (cat. no. 11647229001), according to the manufacturer's protocols. For the MTT assay, 2,000 cells were seeded into 96-well plates. On the next day, $5 \mathrm{mg} / \mathrm{ml}$ MTT solution (GenView SA, Lausanne, Switzerland; cat. no. JT343) was added to each well. Four hours later, $100 \mu \mathrm{l}$ DMSO was added to dissolve formazan particles, with votex for 2-5 min. OD490/570 of each well was read using a microplate spectrophotometer (Tecan Group Ltd., Mannedorf, Switzerland; cat. no. M2009PR). For the BrDU assay, cells were seeded into 96-well plates, and the OD450 of each well was recorded.

Apoptosis assay. Flow cytometry was used to detect the apoptotic rate. AGS cells $\left(5 \times 10^{5}\right)$ cells/well with or without siC1QTNF6 lentivirus infection were plated into 6-well plates and grown to $90 \%$ confluence. Following incubation, the AGS cells were harvested by centrifugation at $179 \mathrm{x} g$ for $5 \mathrm{~min}$ and incubated with $10 \mu \mathrm{l}$ Annexin V-APC for $10-15 \mathrm{~min}$. Next, the cells were analyzed using a flow cytometer (EMD Millipore) and FlowJo software 10.5.2.

Statistical analysis. Fisher's exact test and Student's t-test were used to analyze the relative expression of C1QTNF6 protein in human gastric carcinoma and normal tissues. Two-way analysis of variance with a post hoc Bonferroni's test was used to analyze the relative expression of C1QTNF6 in SGC-7901, AGS, BGC-823 and MGC-803 cell lines, and cell proliferation, migration, invasion and apoptosis. Data are presented as the mean \pm standard error of the mean $(n=3)$. All these experiments were conducted in triplicate. $\mathrm{P}<0.05$ was considered to indicate a statistically significant difference. Results were analyzed using GraphPad 5.0 (GraphPad Software, Inc., La Jolla, CA, USA).

\section{Results}

C1QTNF6 gene expression was upregulated in GC tissues compared with that in peritumoral normal gastric tissues. To investigate the alterations of mRNAs, total RNA samples were isolated from tumor and the peritumoral normal gastric tissues to conduct microarray analysis. Of the $\sim 49,395$ detectable mRNAs, the expression of 238 genes had changed by $>5$ times. The microarray measurements are also displayed as a heat-map matrix (Fig. 1). Compared with the peritumoral normal gastric tissues, the expression of 109 mRNAs 


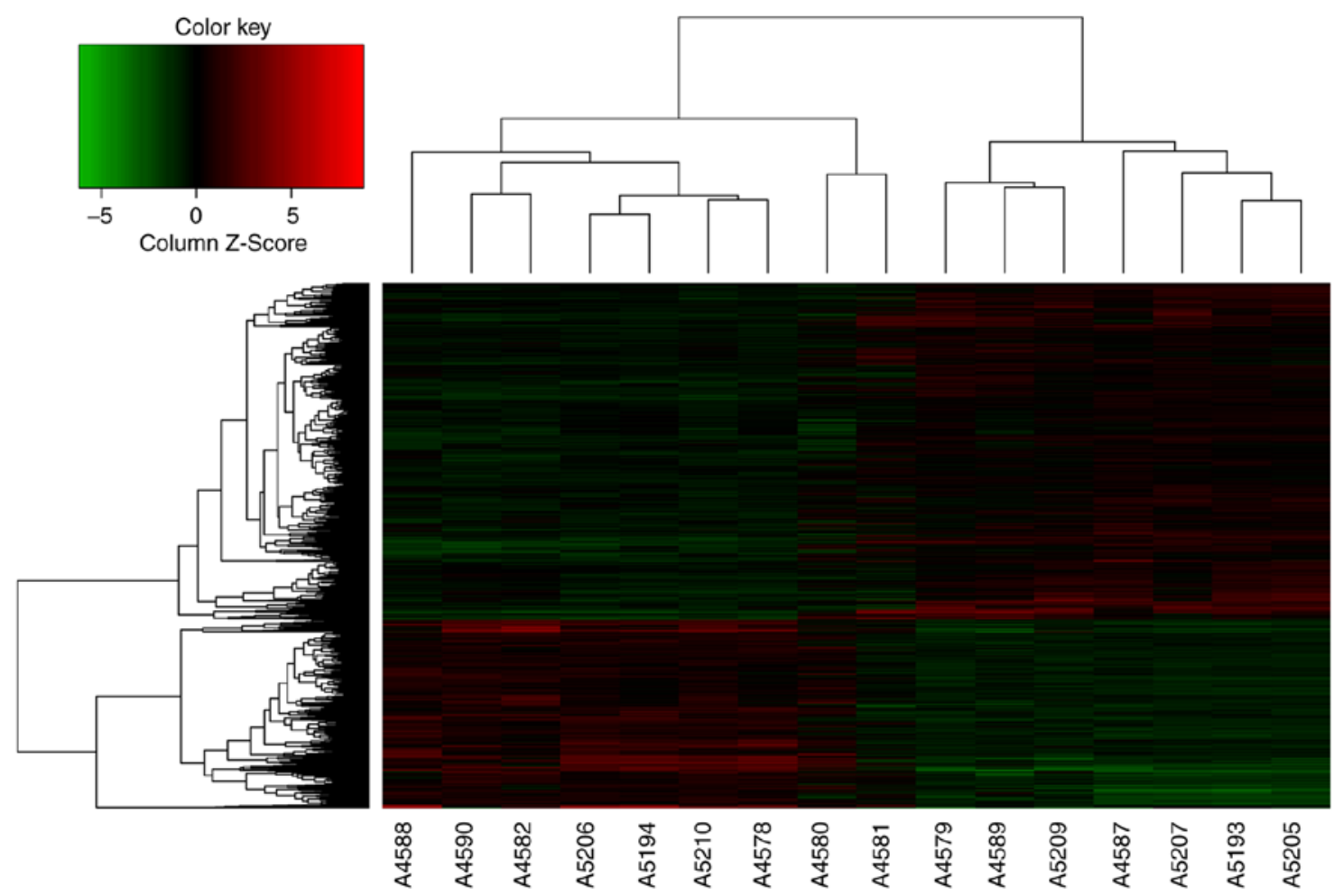

Figure 1. Microarray heatmap in which genes are grouped based on their pattern of gene expression. Green indicates reduced expression, red indicates increased expression.

was upregulated, while the expression of 129 mRNAs was downregulated in GC tissues. C1QTNF6 was significantly upregulated by 1.59 -fold in GC tissues. The influence of the C1QTNF6 gene on GC cell proliferation, migration and invasion in vitro was investigated in the present study.

CIQTNF6 was highly expressed in GC. Next, the present study aimed to determine the expression status of C1QTNF6 in GC. Initially, the protein expression of C1QTNF6 in 52 primary GC samples and paratumoral normal gastric tissues was examined by IHC (Fig. 2A). Notably, the protein expression of C1QTNF6 was observed among GC tissues with different degrees of differentiation, including in poorly- and moderately-differentiated adenocarcinoma tissues. C1QTNF6 was primarily expressed in the cytoplasm and exhibited a diffuse granular distribution. The expression rate of C1QTNF6 [80.7\% (42/52)] in GC tissues was significantly higher than that in the normal gastric tissues [23.1\% (12/52); $\mathrm{P}<0.05$; Fig. 2B]. There was no significant difference in C1QTNF6 expression between GC samples with different pathological grades, depth of infiltration, lymph node metastasis, lymph vascular space involvement or nerve infiltration.

Additionally, the mRNA expression profile of C1QTNF6 was also assessed in GC cell lines. The results revealed high mRNA expression of C1QTNF6 in all four GC cell lines (SGC-7901, AGS, SGC-823 and MGC-803; Fig. 2C). In the subsequent experiments, in order to assess the in vitro effect of C1QTNF6 on cell proliferation, migration, invasion and apoptosis, the AGS cell line was used for C1QTNF6-knockdown.

Protein detection of C1QTNF6-knockdown cell lines. In order to determine whether C1QTNF6 serves a role in the growth and proliferation of GC cells, the present study first established the C1QTNF6-knockdown AGS cell line. RT-qPCR confirmed the efficiency of C1QTNF6-knockdown (Fig. 3A). The expression of C1QTNF6 in knockdown cells was 52.8\% lower than that in the shCtrl cells, which indicated the successful establishment of the C1QTNF6-knockdown cell line. The growth of siC1QTNF6 and shCtrl cells from days 1 to 5 was monitored using Celigo instrument and immunofluorescence microscopy. The fluorescence intensity (Fig. 3B) and cell number (Fig. 3C) of the C1QTNF6-knockdown AGS cell line were weaker than that of the shCtrl cells.

The effect of C1QTNF6 on AGS cell proliferation was further assessed by MTT assay or using the Roche BrDU kit. For the MTT assay, 3 days after the shRNA lentivirus infection, 2,000 cells were seeded into 96-well plates and were allowed to grow for 5 days. The AGS cell proliferation rate was significantly suppressed following C1QTNF6 interference (Fig. 3D). The results of the BrDU assay demonstrated that on the 4th day of culture, the proliferation index of AGS cells was reduced by $\sim 27 \%$ following C1QTNF6-interference (Fig. 3E), which suggested that the proliferative ability of cells was blocked as a result of C1QTNF6-knockdown.

Subsequently, the colony formation ability of the AGS cells was investigated; the results revealed that C1QTNF6-knockdown markedly decreased the colony formation efficiency of AGS cells $(\mathrm{P}=0.00329)$, compared with that of cells transfected with negative control plasmid (Fig. 3F).

ClQTNF6-knockdown inhibits AGS cell invasion and migration. To detect whether C1QTNF6 affects the cell invasion ability, a Transwell chamber migration assay was conducted using AGS cells infected with siC1QTNF6 or 
A
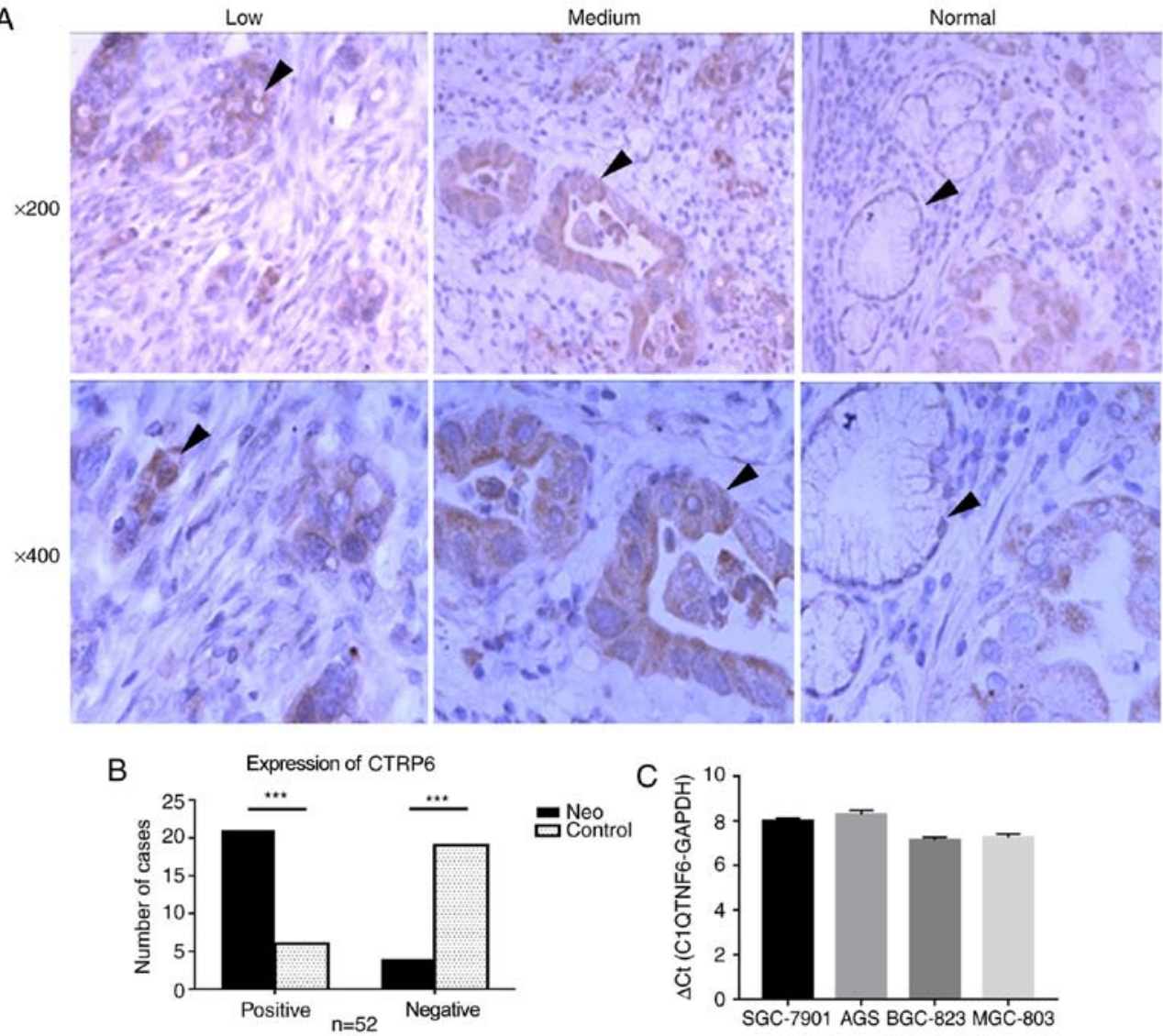

Figure 2. C1QTNF6 expression in GC tissues and GC cell lines. Fifty-two GC samples and the paratumoral normal gastric tissues were isolated from fresh specimens. (A) Immunohistochemical analysis of C1QTNF6 protein expression in GC samples and the peritumoral normal gastric tissues. Magnification, $\mathrm{x} 200$ and $\mathrm{x} 400$. (B) The expression of C1QTNF6 in GC tissues and normal tissues $\left({ }^{* * *} \mathrm{P}<0.001\right.$ vs. Control group). (C) Expression levels of C1QTNF6 mRNA in GC SGC-7901, AGS, SGC-823 and MGC-803 cell lines as determined by reverse transcription-quantitative polymerase chain reaction. Data are presented as the mean \pm standard error of the mean $(n=3)$. GC, gastric carcinoma.

siCtrl lentivirus. As demonstrated in Fig. 4A, infection with siC1QTNF6 notably decreased the invasive ability of AGS cells. The inhibitory rate of siC1QTNF6 on AGS cell invasion was $\sim 76 \%$ (Fig. 4B).

Wound-healing assay revealed that the migration ability of C1QTNF6-knockdown cells at 8 and $24 \mathrm{~h}$ of culture was decreased by $\sim 52.33 \%(\mathrm{P}=0.0001)$ and $\sim 40 \%(\mathrm{P}=0.003)$, respectively (Fig. $4 \mathrm{C}$ and $\mathrm{D}$ ); these results indicated a significant difference between the shCtrl and the siC1QTNF6 groups.

C1QTNF6-knockdown inhibits the AGS cell cycle. To investigate whether cell cycle alteration leads to decreased proliferation of siC1QTNF6 AGS cells, the cell cycle distribution was detected using a FACScan flow cytometer. Following infection with lentivirus for 5 days, there were fewer AGS cells in the $S$ phase and more cells in the G2/M phase following C1QTNF6-knockdown (Fig. 5A and B). No significant difference was observed between the siC1QTNF6 and shCtrl groups with respect to the distribution of cells in the $G_{1}$ phase, which suggested that the C1QTNF6 gene is associated with AGS cell cycle distribution.

C1QTNF6-knockdown promotes AGS cell apoptosis. In order to test the effect of C1QTNF6 on AGS cell apoptosis, flow cytometry was used to detect the apoptotic rate. Five days after transfection with lentivirus, more early apoptotic cells were observed in the siC1QTNF6 AGS group, compared with that in the shCtrl group (Fig. 6A). The apoptotic rate of siC1QTNF6 AGS cells was increased by $\sim 62 \%$, compared with that of the shCtrl cells (Fig. 6B), which suggested that the C1QTNF6 gene is significantly associated with AGS cell apoptosis.

\section{Discussion}

A previous study demonstrated that, under physiological conditions, CTRPs function as molecular mediators connecting inflammatory and metabolic diseases (18). In addition, CTRPs also function in tumor tissues. C1QTNF6 was revealed to be highly expressed in hepatocellular carcinoma (12), and associated with clinicopathological parameters, which suggested that C1QTNF6 is likely to be a diagnostic biomarker for hepatocellular carcinoma. Furthermore, CTRP8-induced human glioma cell migration can be inhibited by a small competitor peptide derived from C1QTNF6 (11). Taken together, these findings suggested an important role of C1QTNF6 in physiological and pathological states. The present study reported high expression levels of C1QTNF6 in GC, and C1QTNF6 was revealed to promote the proliferation and migration of GC cells in vitro and to serve a role in cell apoptosis. These results provided crucial evidence for further studies on reproduction and metastasis of GC cells in vivo. The present study provided novel evidence for 

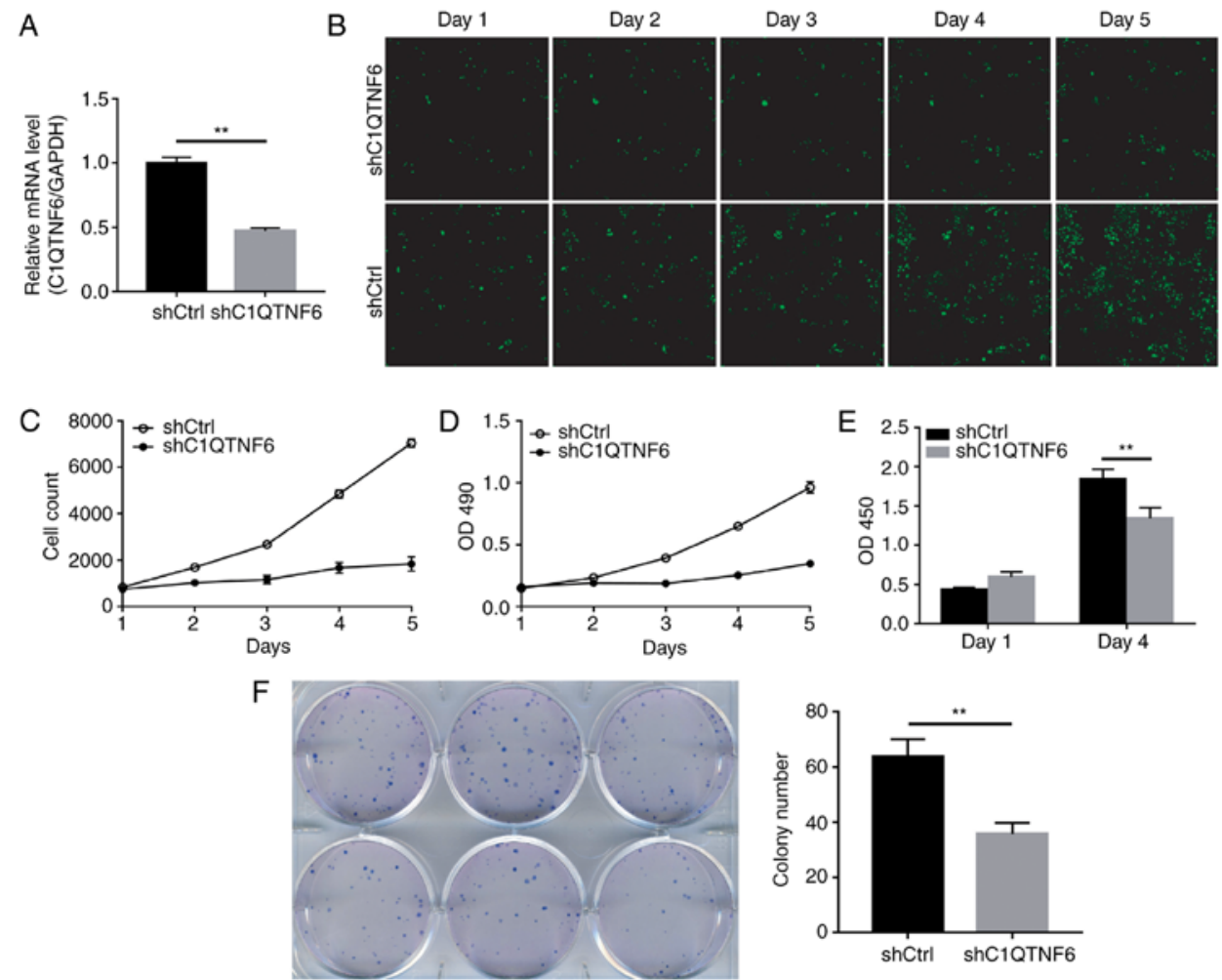

Figure 3. Effects of siRNA-C1QTNF6 on the proliferation of AGS cells. (A) Reverse transcription-quantitative polymerase chain reaction analysis of siRNA-knockdown efficiency. Data are presented as the mean \pm SEM $(n=3),{ }^{* * *} \mathrm{P}<0.01$ vs. shCtrl. (B) The fluorescence intensity (magnification, $\left.x 200\right)$ and (C) cell number of the siC1QTNF6 and shCtrl cells grown from days 1 to 5. (D) The AGS cell proliferation rate of siC1QTNF6 and shCtrl cells, as indicated by the MTT assay. (E) The proliferation index of the siC1QTNF6 and shCtrl cells, as indicated by the BrDU assay. Data are presented as the mean \pm SEM ( $=3$ ); ${ }^{* *} \mathrm{P}<0.01$ vs. shCtrl. (F) Colony formation efficiency of AGS cells with C1QTNF6-knockdown. Data are presented as the mean $\pm \mathrm{SEM}(\mathrm{n}=3)$; ${ }^{* * *} \mathrm{P}<0.01 \mathrm{vs}$. shCtrl. siRNA, small interfering RNA; shRNA, short hairpin RNA; Ctrl, control; SEM, standard error of the mean.

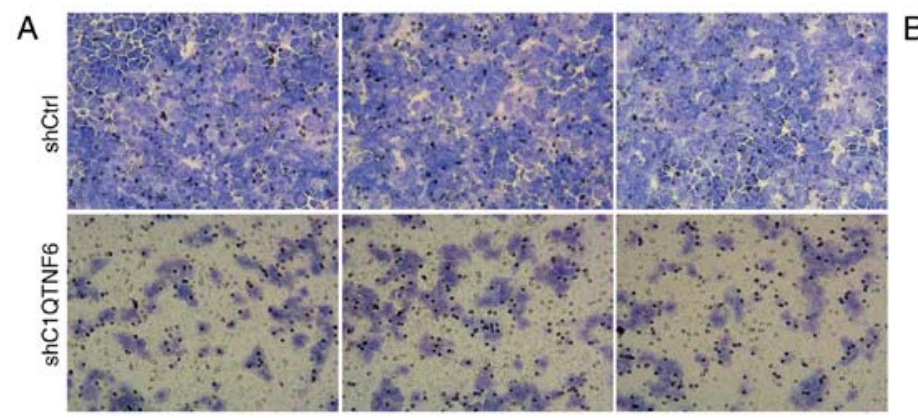

\section{B}
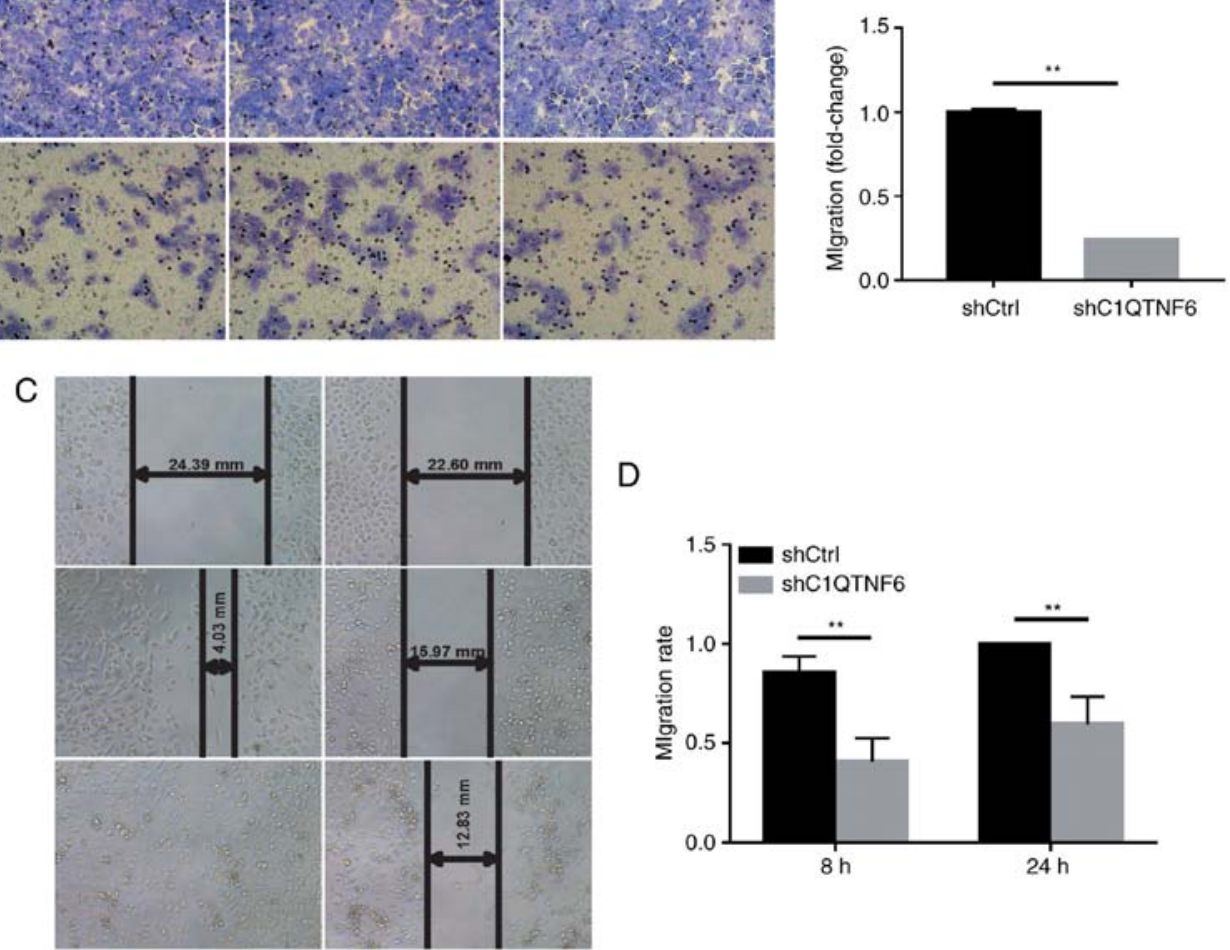

D

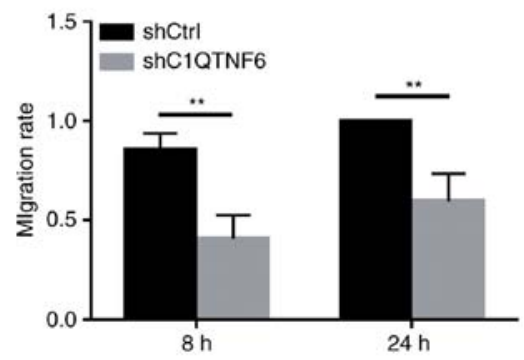

Figure 4. Effects of siRNA-C1QTNF6 on AGS cell invasion and migration. (A) Transwell chamber invasion assay of AGS cells infected with siC1QTNF6 or siCtrl lentivirus (magnification, $\mathrm{x} 200$ ). (B) The inhibitory rates of siC1QTNF6 on AGS cell invasion. Data are presented as the mean $\pm \mathrm{SEM}(\mathrm{n}=3)$; ${ }^{* * *} \mathrm{P}<0.01 \mathrm{vs.}$ shCtrl. (C) Photographs of wound healing assay obtained at 0,8 and $24 \mathrm{~h}$ after scratching. (D) The graph of distance of wound closure. Data are presented as the mean \pm SEM $(n=3) ;{ }^{* *} \mathrm{P}<0.01$ vs. shCtrl. siRNA, small interfering RNA; shRNA, short hairpin RNA; Ctrl, control; SEM, standard error of the mean. 

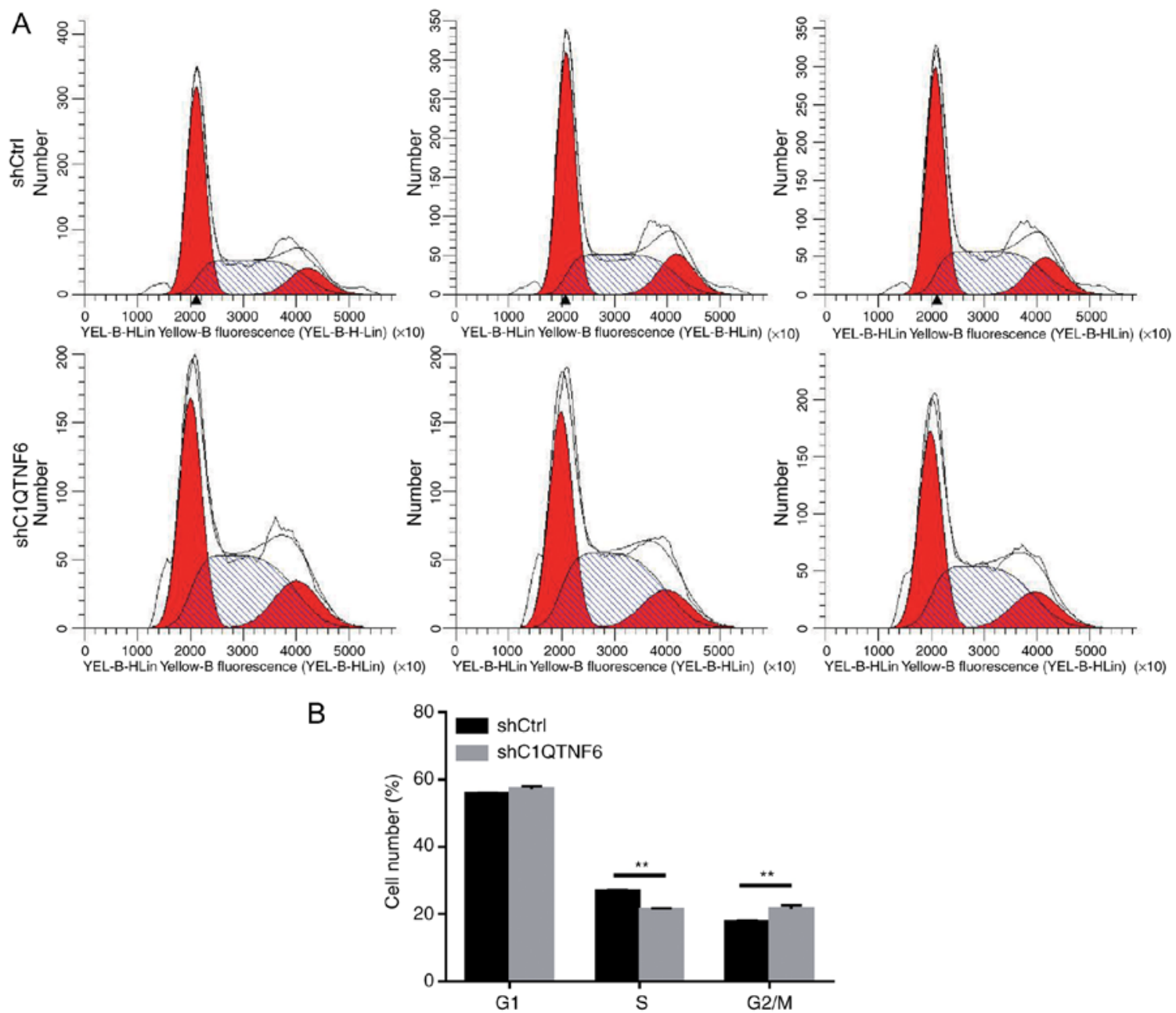

Figure 5. Effects of siRNA-C1QTNF6 on AGS cell cycle. (A) The cell cycle distributions of the siC1QTNF6 and control cells detected using a FACScan flow cytometer. (B) The cartograms of corresponding images. Data are presented as the mean \pm standard error of the mean $(\mathrm{n}=3) ;{ }^{* *} \mathrm{P}<0.01 \mathrm{vs}$. shCtrl. siRNA, small interfering RNA.
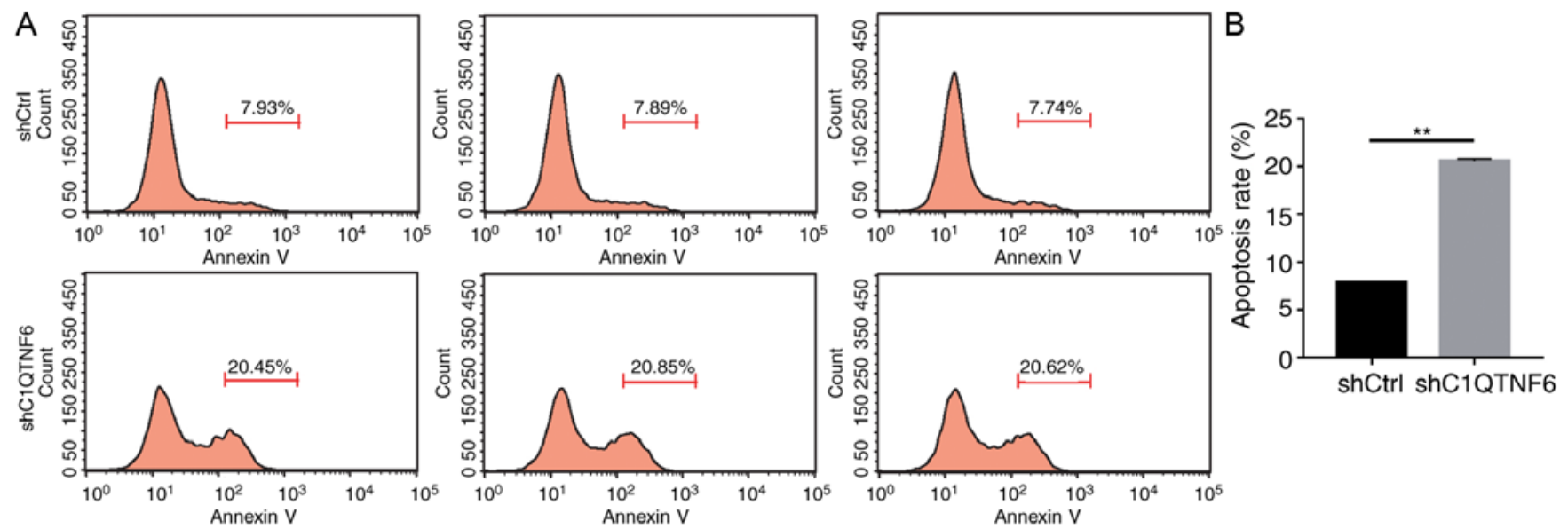

Figure 6. Effects of siRNA-C1QTNF6 on apoptosis of AGS cells. C1QTNF6-knockdown increases the early apoptotic rate of AGS cells. (A) Representative images of flow cytometry. (B) The cartograms of corresponding images. Data are presented as the mean \pm standard error of the mean $(\mathrm{n}=3)$; ${ }^{* *} \mathrm{P}<0.01 \mathrm{vs}$. shCtrl. siRNA, small interfering.

the design of small molecular drugs targeting C1QTNF6 to treat GC. Furthermore, microarray assay was conducted, and numerous differentially expressed proteins were detected, which established the basis to determine the molecular mechanism of C1QTNF6 in the proliferation and migration of GC cells.

The present study demonstrated that C1QTNF6 expression in GC tissues and cell lines has an impact on cell proliferation, 
migration and invasion, which suggested that alternative expression of C1QTNF6 altered cancer cell life processes. However, Wang et al (19) demonstrated that downregulation of C1QTNF6 mRNA contributed toward invasiveness of the human breast cancer MCF-7 cell line. In this aforementioned study, C1QTNF6 was revealed to be directly targeted by miR-29b, which may be considered as a tumor promoter or suppressor, depending on the type of cell or tissue. Therefore, C1QTNF6 may also serve different roles in different cell types with respect to cell invasion or migration. In the present study, inhibition of C1QTNF6 induced G2-M cell cycle arrest and cell apoptosis, which suggested that C1QTNF6 sensitizes GC cells to apoptosis.

The GC AGS cells exhibited a notable decrease in wound healing ability following C1QTNF6-knockdown following 8 or $24 \mathrm{~h}$ culture. This may have been caused by inhibition of the expression of the potent anti-inflammatory cytokine, IL10, since C1QTNF6 can induce IL-10 expression in Raw264.7 cells (20). IL-10 was revealed to enhance wound healing of cutaneous flaps following ischemia reperfusion injury (21), and to promote regenerative healing in an adult model of scar formation (22). Additionally, numerous other factors may also be involved in regulating C1QTNF6-mediated wound healing.

The human C1QTNF family has 16 members that consist of 4 distinct domains, i.e., an N-terminal signal peptide, a short variable domain, a collagen-like domain, and a C-terminal Clq-like globulardomain (23-25). A number of protein members are involved in the regulation of cell apoptosis. Administration of CTRP9 was demonstrated to attenuate cardiomyocyte apoptosis following acute myocardial infarction (26) or myocardial ischemia reperfusion (27), and to decrease the apoptotic rate of primary pulmonary artery epithelial cells due to pulmonary arterial hypertension (28). CTRP3 protected bone marrow derived mesenchymal stem cells from hypoxia-induced apoptosis through the phosphoinositide 3-kinase/protein kinase B signaling pathway (29). CTRP3 also serves a protective role in cardiac infarction through its anti-apoptotic effect in cardiomyocytes (30). CTRP4 was demonstrated to protect against apoptosis induced by chemotherapeutics and thereby promote tumor cell survival (3). The present study was the first to report the anti-apoptotic effect of C1QTNF6 in GC AGS cells; the results are consistent with those of previous studies (28-30), which suggested that C1QTNF family proteins primarily perform cytoprotective and anti-apoptotic functions. In addition, the results of the present study may be applied to a C1QTNF6-knockout animal model to extend and confirm the results derived from a cell culture model. A limitation of the present study is that the cellular protection mechanism of C1QTNF6 remains unclear. Further transcriptomic analysis in GC cells following inhibition of C1QTNF6 expression may provide further insight into the anti-apoptotic mechanism of this protein.

In conclusion, the imbalance of cell proliferation and apoptosis may contribute toward tumorigenesis, and changes in cell motility may lead to metastasis of cancer cells. C1QTNF6 affects the proliferation, migration, invasion and apoptosis of GC cells. With respect to the association between GC and C1QTNF6, the present study demonstrated the following: i) The C1QTNF6 gene is upregulated in GC tissues and cell lines; and ii) interference of C1QTNF6 expression influenced the proliferation, migration and invasion ability of GC cells in vitro. Therefore, the results of the present study provided novel evidence for GC therapy.

\section{Acknowledgements}

Not applicable.

\section{Funding}

The present study was supported by grants from Qingdao Municipal Science and Technology Bureau (grant no. 17-3-3-35-nsh).

\section{Availability of data and materials}

The datasets generated and/or analyzed during the present study are available from the corresponding author on reasonable request.

\section{Authors' contributions}

X-JJ, DW and H-XQ designed the study. H-XQ, LC, X-YM, Z-JW, Y-XC and Y-PY collected and analyzed the data. H-XQ, LC and X-YM drafted and wrote the manuscript. X-JJ and DW revised the manuscript critically for intellectual content. All authors gave intellectual input to the study and approved the final version of the manuscript.

\section{Ethics approval and consent to participate}

The Institutional Review Board of Qingdao Municipal Hospital approved the study protocol. All procedures involving human participants were performed in accordance with the ethical standards of the Institutional and National Research Committee, and the 1964 Declaration of Helsinki and its later amendments or comparable ethical standards. Written informed consent was obtained from all patients.

\section{Patient consent for publication}

Written informed consent was obtained from all patients.

\section{Competing interests}

The authors declare that they have no competing interests.

\section{References}

1. Chung HW and Lim JB: Role of the tumor microenvironment in the pathogenesis of gastric carcinoma. World J Gastroenterol 20: 1667-1680, 2014.

2. Qiu MZ and Xu RH: The progress of targeted therapy in advanced gastric cancer. Biomark Res 1: 32, 2013.

3. Li Q, Wang L, Tan W, Peng Z, Luo Y, Zhang Y, Zhang G, Na D, Jin P, Shi T, et al: Identification of ClqTNF-related protein 4 as a potential cytokine that stimulates the STAT3 and NF-kappaB pathways and promotes cell survival in human cancer cells. Cancer Lett 308: 203-214, 2011.

4. Akiyama H, Furukawa S, Wakisaka S and Maeda T: CTRP3/cartducin promotes proliferation and migration of endothelial cells. Mol Cell Biochem 304: 243-248, 2007. 
5. Park SY, Choi JH, Ryu HS, Pak YK, Park KS, Lee HK and Lee W: C1q tumor necrosis factor alpha-related protein isoform 5 is increased in mitochondrial DNA-depleted myocytes and activates AMP-activated protein kinase. J Biol Chem 284: 27780-27789, 2009.

6. Peterson JM, Wei Z and Wong GW: C1q/TNF-related protein-3 (CTRP3), a novel adipokine that regulates hepatic glucose output. J Biol Chem 285: 39691-39701, 2010.

7. Chavali VR, Khan NW, Cukras CA, Bartsch DU, Jablonski MM and Ayyagari R: A CTRP5 gene S163R mutation knock-in mouse model for late-onset retinal degeneration. Hum Mol Genet 20: 2000-2014, 2011.

8. Hofmann C, Chen N, Obermeier F, Paul G, Buchler C, Kopp A, Falk W and Schäffler A: C1q/TNF-related protein-3 (CTRP-3) is secreted by visceral adipose tissue and exerts antiinflammatory and antifibrotic effects in primary human colonic fibroblasts. Inflamm Bowel Dis 17: 2462-2471, 2011.

9. Thanasupawat T, Glogowska A, Burg M, Wong GW, Hoang-Vu C, Hombach-Klonisch S and Klonisch T: RXFP1 is targeted by complement C1q tumor necrosis factor-related factor 8 in brain cancer. Front Endocrinol (Lausanne) 6: 127, 2015.

10. Klonisch T, Glogowska A, Thanasupawat T, Burg M, Krcek J, Pitz M, Jaggupilli A, Chelikani P, Wong GW and Hombach-Klonisch S: Structural commonality of C1q TNF-related proteins and their potential to activate relaxin/insulin-like family peptide receptor 1 signalling pathways in cancer cells. Br J Pharmacol 174: 1025-1033, 2017.

11. Glogowska A, Kunanuvat U, Stetefeld J, Patel TR, Thanasupawat T, Krcek J, Weber E, Wong GW, Del Bigio MR, Hoang-Vu C, et al: Clq-tumour necrosis factor-related protein 8 (CTRP8) is a novel interaction partner of relaxin receptor RXFP1 in human brain cancer cells. J Pathol 231: 466-479, 2013.

12. Takeuchi T, Adachi Y and Nagayama T: Expression of a secretory protein ClqTNF6, a ClqTNF family member, in hepatocellular carcinoma. Anal Cell Pathol (Amst) 34: 113-121, 2011.

13. Wu WJ, Mo DL, Zhao CZ, Zhao C, Chen YS, Pang WJ and Yang GS: Knockdown of CTRP6 inhibits adipogenesis via lipogenic marker genes and Erk1/2 signalling pathway. Cell Biol Int 39: 554-562, 2015.

14. Murayama MA, Kakuta S, Inoue A, Umeda N, Yonezawa T, Maruhashi T, Tateishi K, Ishigame H, Yabe R, Ikeda S, et al CTRP6 is an endogenous complement regulator that can effectively treat induced arthritis. Nat Commun 6: 8483, 2015.

15. Fan RH, Zhu XM, Sun YW, Peng HZ, Wu HL and Gao WJ: CTRP6 inhibits fibrogenesis in TGF-beta1-stimulated human dermal fibroblasts. Biochem Biophys Res Commun 475: 356-360, 2016.

16. Livak KJ and Schmittgen TD: Analysis of relative gene expression data using real-time quantitative PCR and the 2(-Delta Delta C(T)) method. Methods 25: 402-408, 2001.

17. Chen Z, He T, Zhao K and Xing C: Anti-metastatic activity of fangchinoline in human gastric cancer AGS cells. Oncol Lett 13: 655-660, 2017.
18. Schaffler A and Buechler C: CTRP family: Linking immunity to metabolism. Trends Endocrinol Metab 23: 194-204, 2012.

19. Wang C, Gao C, Zhuang JL, Ding C and Wang Y: A combined approach identifies three mRNAs that are down-regulated by microRNA-29b and promote invasion ability in the breast cancer cell line MCF-7. J Cancer Res Clin Oncol 138: 2127-2136, 2012.

20. Kim MJ, Lee W, Park EJ and Park SY: ClqTNF-related protein-6 increases the expression of interleukin-10 in macrophages. Mol Cells 30: 59-64, 2010.

21. Hu M, Ludlow D, Alexander JS, McLarty J and Lian T: Improved wound healing of postischemic cutaneous flaps with the use of bone marrow-derived stem cells. Laryngoscope 124: 642-648, 2014.

22. Peranteau WH, Zhang L, Muvarak N, Badillo AT, Radu A, Zoltick PW and Liechty KW: IL-10 overexpression decreases inflammatory mediators and promotes regenerative healing in an adult model of scar formation. J Invest Dermatol 128: 1852-1860, 2008.

23. Ahima RS, Qi Y, Singhal NS, Jackson MB and Scherer PE: Brain adipocytokine action and metabolic regulation. Diabetes 55 (Suppl 2): S145-S154, 2006.

24. Scherer PE, Williams S, Fogliano M, Baldini G and Lodish HF: A novel serum protein similar to Clq, produced exclusively in adipocytes. J Biol Chem 270: 26746-26749, 1995

25. Kopp A, Bala M, Weigert J, Buchler C, Neumeier M, Aslanidis C, Scholmerich $\mathrm{J}$ and Schaffler A: Effects of the new adiponectin paralogous protein CTRP-3 and of LPS on cytokine release from monocytes of patients with type 2 diabetes mellitus. Cytokine 49: $51-57,2010$

26. Sun Y, Yi W, Yuan Y, Lau WB, Yi D, Wang X, Wang Y, Su H, Wang X, Gao E, et al: Clq/tumor necrosis factor-related protein-9, a novel adipocyte-derived cytokine, attenuates adverse remodeling in the ischemic mouse heart via protein kinase A activation. Circulation 128 (11 Suppl 1): S113-S120, 2013.

27. Bai S, Cheng L, Yang Y, Fan C, Zhao D, Qin Z, Feng X, Zhao L, Ma J, Wang X, et al: C1q/TNF-related protein 9 protects diabetic rat heart against ischemia reperfusion injury: Role of endoplasmic reticulum stress. Oxid Med Cell Longev 2016: 1902025,2016

28. Li Y, Geng X, Wang H, Cheng G and Xu S: CTRP9 ameliorates pulmonary arterial hypertension through attenuating inflammation and improving endothelial cell survival and function. J Cardiovasc Pharmacol 67: 394-401, 2016.

29. Hou M, Liu J, Liu F, Liu K and Yu B: Clq tumor necrosis factor-related protein-3 protects mesenchymal stem cells against hypoxia- and serum deprivation-induced apoptosis through the phosphoinositide 3-kinase/Akt pathway. Int J Mol Med 33: 97-104, 2014

30. Yi W, Sun Y, Yuan Y, Lau WB, Zheng Q, Wang X, Wang Y, Shang X, Gao E, Koch WJ, et al: Clq/tumor necrosis factor-related protein-3, a newly identified adipokine, is a novel antiapoptotic, proangiogenic, and cardioprotective molecule in the ischemic mouse heart. Circulation 125: 3159-3169, 2012. 